\title{
Antiepileptic drugs as prophylaxis for postcraniotomy seizures
}

\author{
Nikola Vojvodic ${ }^{1}$, Aleksandar Ristic ${ }^{1}$, and Dragoslav Sokic ${ }^{1}$ \\ ${ }^{1}$ Institute of Neurology, Belgrade, Serbia
}

\begin{abstract}
This is the protocol for a review and there is no abstract. The objectives are as follows:

The objective of this review is to determine the efficacy and safety of AEDs when used prophylactically for people undergoing craniotomy.
\end{abstract}

\section{BACKGROUND}

The incidence of epilepsy following supratentorial surgery for non-traumatic pathology has been estimated to be 15\%-20\% (Foy 1981), but due to the nature of the underlying disease for which surgery is undertaken, the risk of postcraniotomy seizures may vary from $3 \%$ to $92 \%$. The chance of de novo seizures occurring in people who have no history of epilepsy decreases with the passage of time. The highest incidence of postoperative epilepsy is within the first month after cranial surgery (two-thirds of the seizures) (North 1983), and 75\% of those who do develop epilepsy will do so within a year of surgery. Approximately $8 \%$ have their first seizure more than two years after surgery. The risk of seizures for some groups of people is higher; for example for those with an arteriovenous malformation who have had a spontaneous intracerebral haematoma, the overall risk does not fall below $10 \%$ between year two and five after surgery, whilst people who suffered from an abscess continue to run a risk of developing epilepsy (92\%) after five years (Shaw 1991).

The routine prophylactic use of antiepileptic drugs for people undergoing craniotomy has remained controversial. Due to the risk of post-operative seizures, some advocate the prophylactic use of antiepileptic drugs (AEDs) to reduce the risk of developing postoperative seizures. There are some uncontrolled retrospective studies that might support the use of AED treatment (Matthew 1980). There are also some data from pathological studies that suggest that some AEDs could have a neuroprotective action on damaged cerebral cortex (Calabresi 2003).

However, some maintain that AEDs should not be used prophylactically, and should only be administered following at least one seizure. Others argue that early postoperative seizures do

Copyright (@) 2009 The Cochrane Collaboration. Published by John Wiley \& Sons, Ltd.

Contact address: Nikola Vojvodic, Institute of Neurology, dr Subotica st. 6, Belgrade, 11000, Serbia. nikov@eunet.yu.

Editorial group: Cochrane Epilepsy Group.

Publication status and date: Edited (no change to conclusions), published in Issue 1, 2009.

DECLARATIONS OF INTEREST

None known. 
not justify the diagnosis of epilepsy and only late seizures are considered to be truly epilepsy (Manaka 2003).

In order to inform a decision regarding the prophylactic use of antiepileptic drugs for people undergoing craniotomy, we need good evidence regarding any benefits and harms and any tradeoffs between benefit and harm that there might be. Potential benefits include reduced short-term seizure recurrence, reduced long-term epilepsy rates, better surgical outcome and quality of life. Harms include adverse effects and poorer surgical outcome. In this review, we will summarise evidence from randomised controlled trials regarding the prophylactic use of AEDs for people undergoing craniotomy.

\section{OBJECTIVES}

The objective of this review is to determine the efficacy and safety of AEDs when used prophylactically for people undergoing craniotomy.

\section{METHODS}

\section{Criteria for considering studies for this review}

Types of studies-Truly randomised controlled trials, either blinded or unblinded.

Types of participants-People of any age and either gender undergoing a supratentorial or infratentorial craniotomy for either therapeutic or diagnostic reasons.

Types of interventions-Any AEDs given prior to or just after craniotomy versus placebo or no treatment controls.

Types of outcome measures-The main outcomes of interest will be:

1. Death from any cause during the treatment study period or during the follow-up study period.

2. Poor functional outcome at the end of the follow-up study period, defined as disability (dependent on others in normal daily activities) or vegetative state.

3. Early seizures (seizures occurring within the first week after craniotomy).

4. Late seizures (seizures after the first week from craniotomy including follow-up period of 1,2 and 5 years postoperatively from craniotomy).

5. Adverse effects of AEDs subdivided into serious (requiring hospitalisation and/or causing death) and those leading to drug withdrawal (e.g. skin rashes; haematological reactions; neuro-behavioural changes and drug intoxication).

If appropriate, we will also analyse the efficacy of the prophylactic treatment within the following subgroups:

i. pathological subgroups;

ii. site of the lesion: 
iii. type of surgery;

iv. presence of different complications after the surgery;

v. different period of treatment commencement (before or after the craniotomy);

vi. people with previous acute symptomatic seizures.

\section{Search methods for identification of studies}

We will identify all relevant clinical trials since 1970, through computerised searches using full MEDLINE with stemming: "Antiepileptic drug and craniotomy and human and prophylaxis". We will also evaluate the EMBASE database, and consult the Cochrane Central Register of Controlled Trials (CENTRAL) in The Cochrane Library.

We will handsearch all major journals in Neurology, Neurosurgery and Epilepsy since 1970 and check them with the Cochrane Epilepsy Group's Specialised Register.

We will contact pharmaceutical companies who manufacture AEDs for further information on trials already gathered and for data on any unpublished trials the reviewers may have overlooked or not found.

We will handsearch the proceedings of the major conferences on epilepsy since 1990.

\section{Data collection and analysis}

One of the review authors will assess articles found for possible inclusion. Two review authors will assess all articles so identified for inclusion. Two review authors will independently extract data from included articles and compare their results. They will resolve disagreements by discussion, and, where necessary, by discussion with a third party. Data to be extracted will include randomisation methods, blinding method (both of treatment administration and of outcome assessment), whether the study authors carried out an intention-to-treat analysis from the published data; whether they could have carried out such an analysis with presented data; and whether they compared treatment groups according to different pathological subgroups and sites of the lesion within and between trials.

In addition, we will extract the major prognostic risk factors for outcome, definition of end points, inclusion and exclusion criteria, type of AEDs used, route and commencement of drug administration and side effects of drugs.

We will also assess the methodological quality of each trial, using a validated scale (Jadad 1996) designed to measure the likelihood of bias in controlled trials based on the main quality pointers: randomisation, double blinding, withdrawals and drop-outs. We will report the quality scores for single trials in the notes section of the "Characteristics of included studies" table. We will not use these scores to weigh results in any way.

The two review authors will extract and cross-check any differing details and will resolve any disagreement by mutual discussion. We will contact the authors of each included trial for further information if we find this to be necessary. 
We will conduct a meta-analysis using The Cochrane Collaboration's Review Manager software for each of these five outcomes. For assessing heterogeneity between trial results, we will use $\mathrm{I}^{2}$ index (Higgins 2002).

\section{WHAT'S NEW}

\begin{tabular}{lll}
\hline Date & Event & Description \\
\hline 20 October 2008 & Amended & Next stage expected updated. \\
\hline
\end{tabular}

\section{HISTORY}

Protocol first published: Issue 3, 2008

\section{Additional references}

Calabresi 2003 . Calabresi P, Cupini LM, Centonze D, Pisani F, Bernardi G. Antiepileptic drugs as a possible neuroprotective strategy in brain ischemia. Annals of Neurology. 2003; 53:693-702. [PubMed: 12783414]

Foy 1981 . Foy PM, Copeland GP, Shaw MDM. The incidence of postoperative seizures. Acta Neurochirurgica. 1981; 55:253-64. [PubMed: 7234531]

Higgins 2002 . Higgins JPT, Thompson SG. Quantifying heterogeneity in a meta-analysis. Statistics in Medicine. 2002; 21:1539-58. [PubMed: 12111919]

Jadad 1996 . Jadad AR, Moore RA, Carroll D, Jenkinson C, Reynolds DJ, Gavaghan DJ, et al. Assessing the quality of reports of randomized clinical trials: is blinding necessary? Controlled Clinical Trials. 1996; 17(1):1-12. [PubMed: 8721797]

Manaka 2003 . Manaka S, Ishijima B, Mayanagi Y. Postoperative seizures: epidemiology, pathology and prophylaxis. Neurologia Medico-Chirurfica (Tokyo). 2003; 43:589-600.

Matthew 1980 . Matthew E, Sherwin AL, Welner SA, Odusote K, Stratford JG. Seizures following intracranial surgery: incidence in the first post-operative week. The Canadian Journal of Neurological Sciences. 1980; 7(4):285-90. [PubMed: 7214242]

North 1983 . North JB, Penhall RK, Hanieh A, Frewin DB, Taylor WB. Phenytoin and postoperative epilepsy: A double-blind study. Journal of Neurosurgery. 1983; 58(5):72-7.

Shaw 1991 . Shaw MDM, Foy PM. Epilepsy after craniotomy and the place of prophylactic anticonvulsant drugs: discussion paper. Journal of the Royal Society of Medicine. 1991; 84(4): 221-3. [PubMed: 2027149]

* Indicates the major publication for the study 